\title{
Improved maximal strength is not associated with improvements in sprint time or jump height in high-level female football players: a cluster-randomized controlled trial
}

\author{
Sigurd Pedersen $^{1 *}\left(\mathbb{D}\right.$, Kim Arne Heitmann ${ }^{1}$, Edvard H. Sagelv', Dag Johansen ${ }^{2}$ and Svein Arne Pettersen ${ }^{1}$
}

\begin{abstract}
Background: Maximal strength increments are reported to result in improvements in sprint speed and jump height in elite male football players. Although similar effects are expected in females, this is yet to be elucidated. The aim of this study was to examine the effect of maximal strength training on sprint speed and jump height in high-level female football players.

Methods: Two female football teams were team-cluster-randomized to a training group (TG) performing maximal strength training (MST) twice a week for 5 weeks, or control group (CG) doing their regular pre-season preparations. The MST consisted of 3-4 sets of 4-6 repetitions at $\geq 85 \%$ of 1 repetitions maximum (1RM) in a squat exercise. Sprint speed and jump height were assessed in 5-, 10- and $15 \mathrm{~m}$ sprints and a counter-movement jump (CMJ) test, respectively. Nineteen participants in TG (18.3 \pm 2.7 years) and 14 in CG (18.3 \pm 2.4 years) completed pre- and posttests and were carried forward for final analyses.

Results: There was no improvement in neither of the sprint times $(p>0.36)$, nor jump height $(p=0.87)$. The players increased their 1RM in squats (main of effect of time: $p<0.00, p n^{2}=0.704$ ), and an interaction effect of time $x$ group was observed $\left(p<0.00, p \eta^{2}=0.516\right)$ where the TG increased their 1RM more than the $C T$ (between subjects effects: $\left.p<0.001, \eta^{2}=0.965\right)$.

Conclusions: MST improved maximal strength in female football players to a large extent; however, the improvement in maximal strength did not result in any transference to sprint speed or jump height.

Trial registration: This study was registered at clinicaltrials.gov PRS (Protocol registration and results System) with the code NCT04048928, 07.08.2019, retrospectively registered.
\end{abstract}

Keywords: Soccer, Sprint, Counter movement jump, 1RM, Squats

\footnotetext{
* Correspondence: Sigurd.pedersen@uit.no

${ }^{1}$ School of Sports Sciences, Faculty of Health Sciences, UiT The Arctic

University of Norway, Tromsø, Norway

Full list of author information is available at the end of the article
}

(c) The Author(s). 2019 Open Access This article is distributed under the terms of the Creative Commons Attribution 4.0 International License (http://creativecommons.org/licenses/by/4.0/), which permits unrestricted use, distribution, and reproduction in any medium, provided you give appropriate credit to the original author(s) and the source, provide a link to the Creative Commons license, and indicate if changes were made. The Creative Commons Public Domain Dedication waiver (http://creativecommons.org/publicdomain/zero/1.0/) applies to the data made available in this article, unless otherwise stated. 


\section{Background}

The intermittent nature of football demands complex physiological taxations [1]. Sprint performance seems to be an important factor, which discriminates between competitive level of players where elite female football players sprint faster compared with lower level players [2, 3]. Over the course of a football match, elite female football players sprint $\left(\geq 25.1 \mathrm{~km} \cdot \mathrm{h}^{-1}\right) \sim 200 \mathrm{~m}$, distributed in $\sim 30$ bouts, of which $95 \%$ are sprints under $10 \mathrm{~m}$ [4], and interestingly, the speed of the sprints has increased for female football players over the last two decades [5], emphasizing the growing importance of sprints in female football.

Approaches for improvements in sprint are many, including sprint training, explosive movements and strength training (ST) [6-8]. Traditionally, ST regimes for developing speed and explosiveness have mainly consisted of repetitions of high velocities and low loads [9]. However, training regimes consisting of training with high loads and low velocity repetitions, usually between 3 and 5 sets of $4-6$ repetitions $\geq 85 \%$ one repetition maximum (1RM) has emerged as a supplement and/or replacement to the low-load high velocity training [10]. The high load low velocity strength training, usually expressed as maximal strength training (MST), is effective for improving maximal strength [11], and may also result in improvements in muscle power and rate of force development in male football players [12]. The rationale behind this training modality for improvements in explosive actions builds on the significant relationship between $1 \mathrm{RM}$ and movement velocity, sprint performance and jump height $[13,14]$. In contrast to the principle of training specificity, training of maximal strength in a nonspecific movement tempo combined with the specific movement itself, is more effective than just training the fast movement alone [15]. The effect from MST on power actions could be explained by an improved neural drive to the muscles involved $[16,17]$ due to the training being performed with maximal intended velocity combined with a load approaching the upper limits of motor unit recruitment [18].

Moreover, the goal of ST is often increased muscle mass [19], however, when comparing conventional hypertrophy training (60-70\% of $1 \mathrm{RM}, 8-12$ repetitions) with MST, MST is superior concerning gains in both $1 R M$ and rate of force development $[11,20]$. Additionally, an increased muscle mass may be detrimental for sports performance involving endurance, such as football, due to the increased body mass. Thus, improving strength with minimal hypertrophy should be favourable, as this will lead to an increased relative strength. According to Newton's second law of motion, an increased relative strength should improve jump height and sprint speed. Minimal hypertrophy in relation to maximal strength gains is best achieved by ST with high loads and low volume [11, 20-22].

In male football players, studies have reported a favorable effect on both 1RM, sprint and jumping performance following MST [20, 23]. Although females and males possess diverse levels of anabolic hormones, they do in general respond similarly after training interventions in most strength outcomes [24]. However, there are reports of a larger relative increase in females compared to males when the same ST is applied [25]. Further, on the muscle fiber level, heavy resistance training is reported to induce hypertrophy for type IIX fiber cross-sectional area in young males only, when compared with young females [26], indicating potential gender differences in response to ST. The effect on strength, sprint and jump height performance following MST in female football players is still to be elucidated. Thus, the aim of this study was to examine if improvement in maximal strength is associated with improvements in sprint and jump height performance following MST.

\section{Methods}

In this cluster-randomized controlled trial, two football teams (playing at level 2 and 3 in Norway) was invited to participate. The study were conducted during the last part of the pre-season preparations, ending 1 week before first seasonal competition. The training group (TG) performed MST training carried out as free-barbell squats twice a week over 5 weeks in addition to the planned pre-season training, while the control group (CG) was instructed to perform their originally planned pre-season training.

\section{Subjects}

The total sample comprised 46 players aged $15-26$ years, where two separate football teams were cluster-randomized to either TG or CG (Table 1). The two teams played at level two and three in Norway, where level two is a national

Table 1 Characteristics of participants

\begin{tabular}{lll}
\hline & TG $(n=24)$ & CG $(n=22)$ \\
\hline Age (years) & $18 \pm 3$ & $19 \pm 2$ \\
Body mass $(\mathrm{kg})$ & $62 \pm 6$ & $63 \pm 10$ \\
Height $(\mathrm{cm})$ & $167 \pm 6$ & $168 \pm 5$ \\
BMl $\left(\mathrm{Kg} / \mathrm{m}^{2}\right)$ & $22.1 \pm 2$ & $22.3 \pm 3$ \\
$\begin{array}{l}\text { Experience with the squat } \\
\text { exercise }\end{array}$ & \\
$\quad$ None & 3 & 8 \\
$\quad$ Some (< 1 year) & 14 & 9 \\
$\quad$ Much (> 1 year) & 7 & 5 \\
\hline
\end{tabular}

Data are mean \pm standard deviation. TG Training group, CG Control group, BMI Body mass index 
league and level three a regional league in Northern Norway. Inclusion criteria was that the players perceived themselves as injury free and able to complete the strength training. Randomization was carried out using the online tool http://www.randomlist.com/team-generator by the first author. Players were only excluded if having injuries that made strength training, running and jumping unachievable. The players carried out $6.5 \mathrm{~h}$ training per week with their team (Table 2). Four players were injured, two did not complete the required amount of training, one withdrew due to time limitations and five withdrew without providing any reason resulting in 19 participants in TG and 15 in CG that completed both pre- and posttests, and were included in the analyses for training effect (Table 3).

According to the declaration of Helsinki, all participants were fully informed of the potential benefits and risks of the study, both orally and written, before signing an informed consent. For participants under 16 years, both the players and their parents gave their written informed consent. The participants were fully informed of their rights to withdraw from the study at any time without providing any reason. This study was approved by the Norwegian Centre for Research Data for the storage of personal data (Approval reference number: 59063 / 3).

\section{Procedures}

All testing and training sessions were conducted in an exercise training laboratory at Alfheim Stadium, Troms $\varnothing$. Prior to the intervention, the players underwent baseline tests over two test days, with a $72 \mathrm{~h}$ washout period to avoid any detrimental effects from the preceding test day: day 1 ) measurement of body mass and body height, 5-, 10- and $15 \mathrm{~m}$ sprint time and a counter-movement jump (CMJ), day 2) $1 R M$ in a free-barbell squat exercise with partial $90^{\circ}$ knee angle range of motion (ROM).

Prior to the tests, the participants were asked to refrain from heavy training the preceding day, and to arrive in the laboratory well-hydrated. All tests and training sessions started in the afternoon, with the same general warm-up routine: 7 min of self-selected low intensity cycling on an ergometer bike (Pro/Trainer, Wattbike Ltd., Nottingham,

Table 2 Weekly team training for CG and TG prior to inclusion

\begin{tabular}{|c|c|c|}
\hline & CG & TG \\
\hline Sessions (n) & $4-5$ & $4-5$ \\
\hline Passing, technique, finishing, possession $\left(\mathrm{min}^{-1}\right)$ & 60 & 270 \\
\hline High intensity small sided games $\left(\mathrm{min}^{-1}\right)$ & 90 & 90 \\
\hline Running and conditioning $\left(\mathrm{min}^{-1}\right)$ & 90 & 45 \\
\hline Strength, balance and injury prevention $\left(\mathrm{min}^{-1}\right)$ & 90 & 0 \\
\hline Stretching $\left(\mathrm{min}^{-1}\right)$ & 60 & 0 \\
\hline Total training time $\left(\right.$ hours ${ }^{-1}: \mathrm{min}^{-1}$ ) & $6: 30$ & $6: 45$ \\
\hline
\end{tabular}

UK) followed by 7 min low intensity running of self-selected speed on artificial grass.

On day 1 , following the general warm-up and three 15 $\mathrm{m}$ strides on a sprinting field, a 15-m sprint test was carried out. Data were assessed in $5 \mathrm{~m}$ splits by photocells mounted to the floor and walls (ATU-X, IC control AB, Stockholm, Sweden) using single-beam electronic barriers. The within-subject coefficient of variation is $2 \%$ for this measurement [27]. The surface consisted of artificial grass, and the players wore their own running shoes. The sprints started with the players in a static position placing their front foot $30 \mathrm{~cm}$ behind the starting line. A timer was triggered by the participant breaking the initial sensor. The rest interval between the single sprint trials was $180 \mathrm{~s}$ [10]. The fastest sprint time of three trials was carried forward for further analyses.

Thereafter, the players rested for 5 min prior to performing the CMJ test [23]. CMJ was assessed by a portable force platform (Hur-Labs, ALU4, Finland), with a validity within $1 \mathrm{~cm}(2 \%)$ when compared with the gold standard mounted floor force platform [28], and a within-subject coefficient of variation of 2.8\% [29]. Force data were recorded by a software (Force platform software suite, HURlabs oy, Kokkola, Finland). This device records only the vertical ground reaction force at a sampling frequency of $1200 \mathrm{~Hz}$ and jump height is automatically calculated by software applying double integration of the force signal through Simpson's rule of integration. The players were instructed to keep their hands placed on the hips and the feet shoulder-width apart. Each player performed two trials with $a \geq 180 \mathrm{~s}$ rest between sets. The highest jump was carried forward for further analysis. Day 1 was ended with a familiarization trial for the squat exercise with low loads.

On day 2, the players returned to the laboratory for the assessment of maximal strength as 1RM. The session was initiated with the same general warm up routine as mentioned above. An Olympic barbell (T-100G; Eleiko, Halmstad, Sweden) and a suitable rack was applied for testing of $1 \mathrm{RM}$. The $\sim 90^{\circ}$ knee angle of each participant was measured during every repetition using a goniometer, and the players were given an orally "go" when being allowed to start the concentric phase of the lift. Prior to starting their $1 \mathrm{RM}$ attempts, the participants warmed up with 10 repetitions with a low load of $\sim 50 \% 1$ RM (subjectively assessed by the instructor). The starting 1RM attempt was an initial acceptable load decided by the instructor. Each 1RM attempt was carried out by a single repetition, with increasing load of 5-10 kg until they failed to execute the 1RM attempt, which on average was five trials. Each attempt was interspaced by $\geq 180 \mathrm{~s}$ of rest. The within-subject coefficient of variation for squat $1 \mathrm{RM}$ is $2.9 \%$ [30]. 
Table 3 Effect of training on body mass, physical performance measures and strength derivatives (Mean \pm SD)

\begin{tabular}{|c|c|c|c|c|c|}
\hline \multirow[t]{2}{*}{ Variables } & \multicolumn{2}{|l|}{$\mathrm{TG}(n=19)$} & \multicolumn{2}{|l|}{ CG $(n=15)$} & \multirow{2}{*}{$\begin{array}{l}P \text { - } \\
\text { value* }\end{array}$} \\
\hline & Pre & Post & Pre & Post & \\
\hline Body mass (kg) & $61.67 \pm 5.40$ & $62.18 \pm 5.31$ & $62.92 \pm 10.48$ & $64.09 \pm 10.49$ & 0.13 \\
\hline \multicolumn{6}{|l|}{ Sprint time (s) } \\
\hline $5 \mathrm{~m}$ & $1.06 \pm 0.05$ & $1.05 \pm 0.05$ & $1.06 \pm 0.06$ & $1.07 \pm 0.06$ & 0.07 \\
\hline $10 m$ & $1.89 \pm 0.07$ & $1.89 \pm 0.08$ & $1.90 \pm 0.09$ & $1.90 \pm 0.09$ & 0.74 \\
\hline $15 \mathrm{~m}$ & $2.66 \pm 0.10$ & $2.64 \pm 0.12$ & $2.67 \pm 0.13$ & $2.66 \pm 0.13$ & 0.51 \\
\hline CMJ Jump Height (cm) & $27.32 \pm 4.94$ & $27.19 \pm 5.93$ & $25.82 \pm 5.45$ & $26.12 \pm 4.83$ & 0.65 \\
\hline 1RM $90^{\circ}$ squat $(\mathrm{kg})$ & $106 \pm 21$ & $137 \pm 16$ & $118 \pm 28$ & $124 \pm 31$ & 0.00 \\
\hline 1RM $90^{\circ}$ squat $\left(\mathrm{kg} / \mathrm{m}_{\mathrm{b}} \mathrm{kg}^{-1}\right)$ & $1.73 \pm 0.33$ & $2.21 \pm 0.32$ & $1.88 \pm 0.34$ & $1.94 \pm 0.37$ & 0.00 \\
\hline 1RM $90^{\circ}$ squat $\left(\mathrm{kg} / \mathrm{mb}^{-0.67}\right)$ & $6.81 \pm 1.29$ & $8.73 \pm 1.14$ & $7.45 \pm 1.39$ & $7.73 \pm 1.52$ & 0.00 \\
\hline
\end{tabular}

TG Training group, CG Control group, CMJ Counter movement jump, $1 R M 1$ repetition maximum. * $P$-value represents between subjects effect

\section{Training intervention}

The players attended supervised training in the laboratory twice a week for 5 weeks. The training session started with the general warm-up routine described above, before starting the strength training. The program consisted of $90^{\circ}$ squats, carried out in the same way as in the $1 R M$ test. The squat training was initiated with three sessions of three sets of six repetitions, followed by seven sessions of four sets of four repetitions. The repetitions were carried out with a slow eccentric movement followed by maximal mobilization in the concentric phase. One hundred eighty second of recovery was given between each set [23]. The load was initially set at $85 \%$ of pre-test $1 \mathrm{RM}$, which the participants increased with $2.5-10 \mathrm{~kg}$ if they could manage more than six or four repetitions, depending on their scheduled program, resulting in a consistent overload during the whole intervention (Fig. 1). Weight lifted for each repetition was logged continuously during the study. Additionally, for ethical reasons, in order to avoid hamstring strains due to an anticipated large agonist-antagonist strength ratio following the intervention, three sets of six repetitions of the Nordic hamstring exercise were performed after the squat exercise for each session with $a \geq 180 \mathrm{~s}$ rest period between sets [31] (Fig. 1).

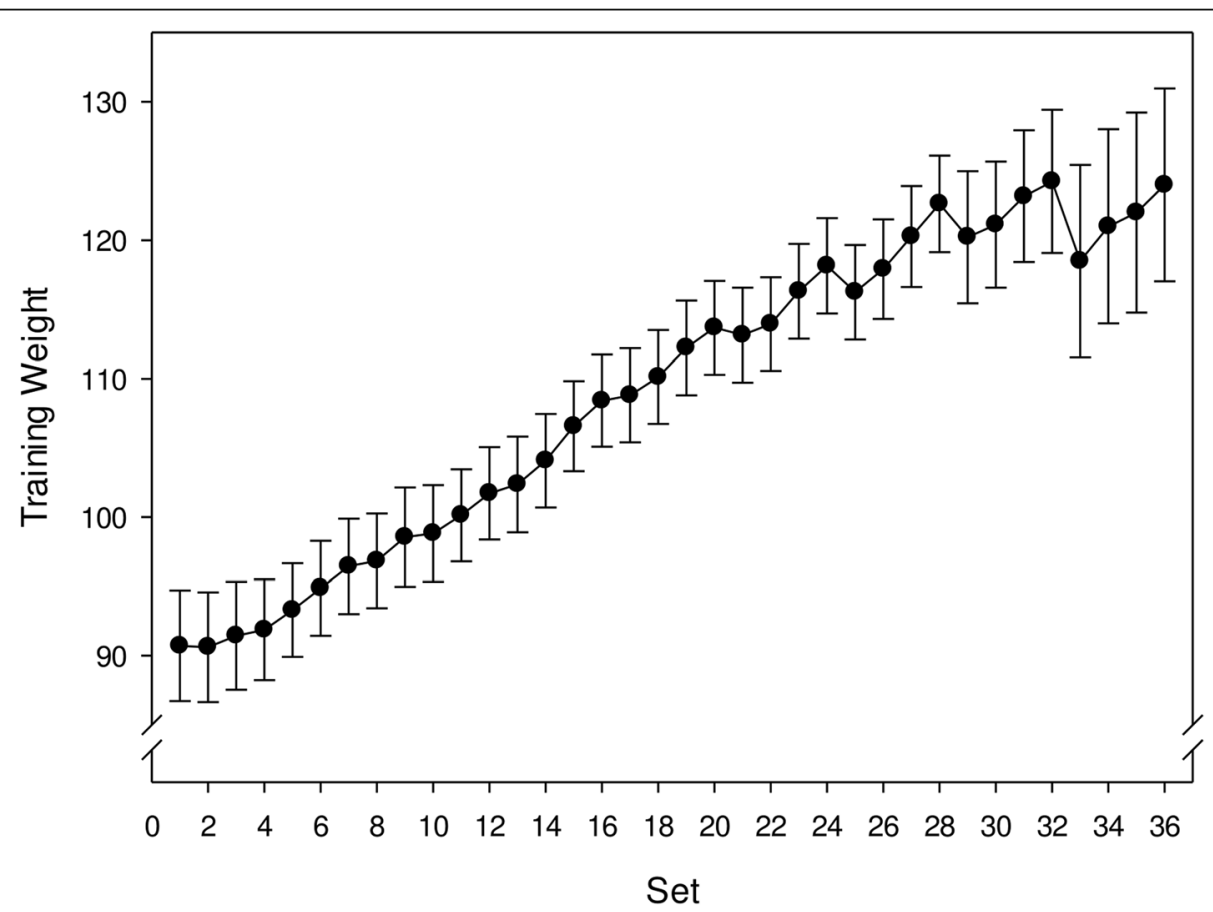

Fig. 1 The logged training for the $90^{\circ}$ squat exercise performed as maximal strength training (MST) by the training group. The dots represent the average weight lifted \pm SE (vertical bars) during each set 


\section{Statistical analyses}

All statistical analyses were carried out using the Statistical Package for Social Sciences (SPSS, Version 25, IBM, USA). The Shapiro Wilk test confirmed all data, except for body weight in CG ( $p=0.02)$ and $5 \mathrm{~m}$ sprint in TG ( $\mathrm{p}=0.02)$, to not deviate from normal distribution. Data were analysed via a two $\mathrm{x}$ two repeated measure analysis of variance (ANOVA). Two levels corresponding to the groups (i.e., TG and CG) are specified as the between-subjects factor. The within-subjects factor (time of test) represents the preand post-tests. Effect sizes were calculated as partial eta squared $\left({ }_{\mathrm{p}} \eta^{2}\right)$ were a small, medium and large effect size was determined as $0.01-0.05,0.06-0.13$ and $\geq 0.14{ }_{\mathrm{p}} \eta^{2}$ [32]. All values are presented as mean \pm standard deviation (SD). Descriptive values for female football players in $90^{\circ}$ squat is reported once $(112 \mathrm{~kg}$ ) [33], where SD is not reported. In one similar study in males where mean $1 \mathrm{RM}$ squat strength is $116 \mathrm{~kg}$, the reported SD was 20.1 [23] $\mathrm{kg}$. It is previously reported that a $24 \%$ increase in 1 RM squat strength results in a $2 \%$ improved sprint performance in male football players [8]. Thus, to observe a $24 \%$ improvement in strength with $80 \%$ power and an alpha level of $0.05,9$ participants are required in each group.

\section{Results}

Nineteen participants in the TG and 15 participants in the CG performed all pre and post-tests and $\geq 70 \%$ of all training sessions (one subject performed $70 \%$, seven subjects performed $80 \%$, five subjects performed $90 \%$ and six subjects performed 100\%). The baseline values for the participants included in the intervention analysis were not different between the two groups (Table 3).

There was no main effect of time for $5 \mathrm{~m}(p=0.77$, $\left.\mathrm{p} \eta^{2}=0.003\right), 10 \mathrm{~m}\left(p=0.82,{ }_{\mathrm{p}} \eta^{2}=0.002\right)$ or $15 \mathrm{~m}(p=$ $\left.0.36, \mathrm{p} \eta^{2}=0.026\right)$ sprint time, and consequently no interaction effects of time $\mathrm{x}$ group was observed $(5 \mathrm{~m}$ : $p=0.72,{ }_{\mathrm{p}} \eta^{2}=0.097,10 \mathrm{~m}: \quad p=0.74, \mathrm{p}^{2}=0.003,15$ : $\left.p=0.51,{ }_{\mathrm{p}} \eta^{2}=0.014\right)$ (Table 3).

Similarly, no main effect of time for CMJ was observed $\left(p=0.87,{ }_{\mathrm{p}} \eta^{2}=0.001\right)$, and consequently no interaction effect of time $\mathrm{x}$ group was observed $\left(p=0.65,{ }_{\mathrm{p}} \eta^{2}=0.006\right)$.

The players increased their 1RM in squats (main of effect of time: $p<0.00,{ }_{\mathrm{p}} \eta^{2}=0.704$ ), and an interaction effect of time $\mathrm{x}$ group was observed $\left(p<0.00,{ }_{\mathrm{p}} \eta^{2}=0.516\right)$ where the TG increased their $1 \mathrm{RM}$ significantly more than the CT (between subjects effects: $p<0.001,{ }_{\mathrm{p}} \eta^{2}=0.965$ ).

The players increased their body mass $(p<0.001$, $\left.{ }_{p} \eta^{2}=0.332\right)$, however, no interaction effect between groups was observed $\left(p=0.13,{ }_{\mathrm{p}} \eta^{2}=0.070\right)$ (Fig. 2).

\section{Discussion}

In this cluster-randomized controlled trial, 5 weeks of MST improved 1RM, but this maximal strength improvement did not result in any improvements in sprint time or $\mathrm{CMJ}$ performance in female football players.

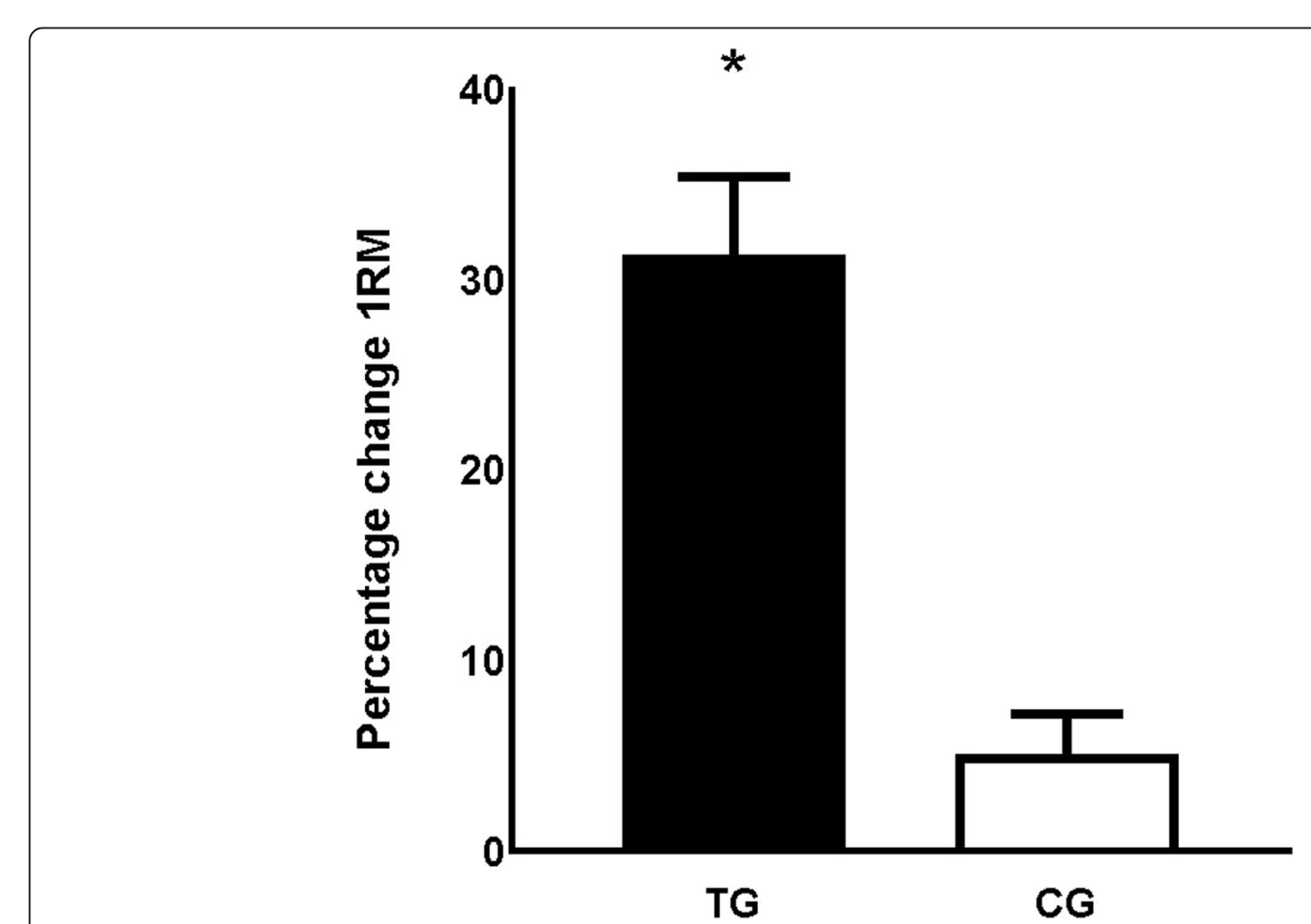

Fig. 2 The mean percentage change from pre- to posttest for $1 \mathrm{RM} \pm \mathrm{SE}$ in $\mathrm{TG}$ and $\mathrm{CG}$. TG = training group; $C \mathrm{G}=$ control group; $1 \mathrm{RM}=1$ repetition maximum. ${ }^{*}=$ Between group difference $p<0.01$ 
We observed a large increase in absolute 1RM strength of $31 \mathrm{~kg}(31 \%)$ for the TG, being highly superior to the 5\% increase in 1RM for the CG. This is a more pronounced increase in strength than observed on average for highly trained male football players [8]. On the one hand, as untrained individuals seem to have greater improvements in strength following ST compared with trained individuals [34], the large improvements in this study may be due to the low experience of ST in the participants of the present study. On the other hand, the baseline values in this study was similar to previously reported values for elite female football players [33], where the present study's participants ended up being considerably stronger than their elite peers (the present study's participants: $137 \mathrm{~kg}$, previously reported values for elite peers: $113 \mathrm{~kg}$ ). Moreover, following 5 weeks of MST, the participants in our study displayed similar absolute $1 \mathrm{RM} 90^{\circ}$ squat strength (present study: $\sim 136 \mathrm{~kg}$ ) as two previously reported elite male football teams (Male players: 116/ $135 \mathrm{~kg}$,) [15, 23].

Previous studies applying ST in female football players have assessed strength outcomes in isometric [35] or isokinetic exercises [36, 37], making comparisons with the present study unattainable as we measured dynamic squat strength [38]. Elite male football players experienced a $52 \%$ increase in absolute 1RM following 16 sessions of MST over 8 weeks [23]. Considering the similar relative increase per training session in the present study $(\sim 3.6 \%$ increase per session) compared with the study by Helgerud et al. [23] $(\sim 3.2 \%$ per session), one may speculate that there are small sex differences in strength improvements following MST in football players. As a linear increase in strength gain has been observed from onset of ST with up to 8 weeks before sign of plateau is observed [39] and duration of our study was 5 weeks, one may speculate whether the players in our study did not reach their expected plateau for strength improvements.

The present study's participants displayed an increase in body mass, which is consistent with earlier findings in men [23]. Considering that both the TG and the CG in the present study performed pre-season training, one explanation for the increased body mass may be an increased water uptake in muscles due to the improved glycogen uptake in muscles [40], which is observed following training initiation [41]. Nevertheless, it is unlikely that the increased body mass solely explains the large increase in $1 \mathrm{RM}$ strength in the present study, suggesting a large improvement in neural drive and/or improved motor unit recruitment following the MST intervention [39].

There were no improvements in sprint following MST in this study, which is in contrast to previous findings in male football players [20, 23]. In fact, an average increase of $23.5 \%$ increase in $1 \mathrm{RM}$ is required for a $2 \%$ improvement in $10 \mathrm{~m}$ sprint in the males [8].
The previous studies conducted in males employed longer intervention duration compared with this present study $[10,20,23]$. Thus, as there may be a dose-response relationship between improvements in explosive actions and ST training duration [42], the intervention duration may have been too short in the present study [43]. However, there are other possible explanations for the lack of improvement in sprint performance. One may be that the players performed insufficiently amounts of specific sprint training in the pre-season cycle. It is previously shown that in order to improve sports-related high-velocity movements, these movements must be performed in everyday training [44]. Moreover, as football is concurrent sport with need for both endurance and strength, an interference effect could be present for the adaptation to training [45]. Interestingly, the interference effect from concurrent training is shown to be more pronounced for adaptations to power actions, compared with adaptations to strength, meaning that force at high velocities is affected to a larger extent than force at low velocities [46], which could explain the lack of improvement in sprint and jump abilities, although strength was increased.

There were no jump height improvements in the present study, which is consistent with a previous study in females [47], but in contrast to a study conducted with males [8]. Previous studies who observed an increased CMJ jump height in female football players included plyometric training [42,48-50], thus, as for sprint adaptations, the specificity of training may explain the unimproved jump performance as well [44].

A stronger muscle will tolerate a higher force, making it more resistant to injury. Although not measured in the current study, ST reduces injury rate, and shortens rehabilitation time [51]. Moreover, the strength of connective tissues and joint stability is also improved after ST [52]. That is of particular importance in the female football player population as they have a two to six times greater prevalence of anterior cruciate ligament injuries compared to their male equivalents [53]. The players in the present study showed similar body mass values as their national level peers [53], which is suggested as optimal for physical performance for female football players [54]. Moreover, as age, anthropometry and physical variables did not differ between the two teams in the present study, comparisons were regarded as appropriate. Finally, although performance by means of sprint speed or jump height was unchanged in the current study, an increased strength could potentially improve performance through pathways not assessed in this paper. For example, this could lead way for future studies with higher ecological value, where the assessment of fatigue delay, technical actions and number of interceptions during match play could be studied following 
such a large increase in strength. MST has mainly been evaluated in male athletes, and this study is to our knowledge the first to investigate the effect of MST for dynamic strength 1RM, sprint speed and jump height in female football players.

There are several limitations of this study. First only six of the participants accomplished $100 \%$ of the training, which means that the other participants had some weeks when they only performed one ST session. Moreover, the CG was instructed to continue their habitual training, without any substitute for the added training time seen in the TG during the intervention. Thus, we are not able to distinguish between the effect of MST and the effect of an added training volume per se.

Although the participants in this study played at senior level football, their mean age was $\sim 18$ years, which can be considered junior level age. In contrast, those who are competing at the national level and in the highest level domestic leagues in other countries, are typically between 20 and 27 years [53]. Thus, although the participants in the present study aim at competing at the highest level possible, they may not be at the peak of their performance level at the present age. Secondly, we propose improved neural adaptations as the main mechanism driving the observed increase in 1RM. However, more sophisticated measurement methods are required to assess which type of neural adaptations that are responsible for this increase.

\section{Conclusions}

MST improves maximal strength in female football players; however, the improvement in maximal strength did not result in any transference to improvements in sprint speed or jump height in this study. This indicates that female football players may need to incorporate specific sprint and jump training into their weekly training routines in order experience improvements in sprint and jump performance. However, our intervention was only 5 weeks, hence, it is unknown whether a longer intervention period would allowed these players to improve their sprint and jump performance, as it is observed previously for male players.

\section{Abbreviations}

1RM: 1 Repetition maximum; CG: Control group; CMJ: Counter movement jump; MST: Maximal strength training; TG: Training group

\section{Acknowledgements}

The authors express thanks to all the study participants for their contribution.

\section{Authors' contributions}

SP: In charge of writing, training, statistics, study design and data collection. ES and KH: training, data collection and manuscript writing. SAP: Study design and manuscript writing. DJ: Manuscript writing. All Authors read and approved the final manuscript.

\section{Funding}

This research was funded by RDA and Tromsø Research Foundation. The funding body had no role in designing the study and data collection, analysis, interpretation, and in writing of the study. The Article Processing Charges were funded by the publication fund at the University Library at UiT the Arctic University of Norway. The authors declare that they have no conflict of interests.

\section{Availability of data and materials}

Trial protocol and the datasets used in in the current study are available from the corresponding author on reasonable request.

\section{Ethics approval and consent to participate}

According to the declaration of Helsinki, all participants were fully informed of the potential benefits and risks of the study, both orally and written, before signing an informed consent. For participants under 16 years, both the players and their parents gave their written informed consent. The participants were fully informed of their rights to withdraw from the study at any time without providing any reason. This study was approved by the Norwegian Centre for Research Data for the storage of personal data (Approval reference number: 59063 / 3). The study adheres to CONSORT guidelines.

\section{Consent for publication}

Not applicable

\section{Competing interests}

The authors declare that they have no competing interests.

\section{Author details}

${ }^{1}$ School of Sports Sciences, Faculty of Health Sciences, UiT The Arctic University of Norway, Tromsø, Norway. ${ }^{2}$ Department of Computer Sciences, Faculty of Natural Sciences and Technology, UiT The Arctic University of Norway, Tromsø, Norway.

Received: 12 April 2019 Accepted: 27 August 2019

Published online: 17 September 2019

\section{References}

1. Morgans R, Orme P, Anderson L, Drust B. Principles and practices of training for soccer. J Sport Health Sci. 2014;3(4):251-7.

2. Haugen TA, Tonnessen E, Seiler S. Anaerobic performance testing of professional soccer players 1995-2010. Int J Sports Physiol Perform. 2013; 8(2):148-56.

3. Vescovi JD. Sprint speed characteristics of high-level American female soccer players: female athletes in motion (FAiM) study. J Sci Med Sport. 2012;15(5):474-8.

4. Datson N, Drust B, Weston M, Jarman IH, Lisboa PJ, Gregson W. Match physical performance of elite female soccer players during international competition. J Strength Cond Res. 2017;31(9):2379-87.

5. Haugen TA, Tonnessen E, Seiler S. Speed and countermovement-jump characteristics of elite female soccer players, 1995-2010. Int J Sports Physiol Perform. 2012;7(4):340-9.

6. Spinks CD, Murphy AJ, Spinks WL, Lockie RG. The effects of resisted sprint training on acceleration performance and kinematics in soccer, rugby union, and Australian football players. J Strength Cond Res. 2007;21(1):77-85.

7. Sedano S, Matheu A, Redondo JC, Cuadrado G. Effects of plyometric training on explosive strength, acceleration capacity and kicking speed in young elite soccer players. J Sports Med Phys Fitness. 2011;51(1):50-8.

8. Silva JR, Nassis GP, Rebelo A. Strength training in soccer with a specific focus on highly trained players. Sports Med Open. 2015;1 (1):17.

9. Buchheit M, Mendez-Villanueva A, Delhomel G, Brughelli M, Ahmaidi S. Improving repeated sprint ability in young elite soccer players: repeated shuttle sprints vs. explosive strength training. J Strength Cond Res. 2010; 24(10):2715-22.

10. Ronnestad BR, Kvamme NH, Sunde A, Raastad T. Short-term effects of strength and plyometric training on sprint and jump performance in professional soccer players. J Strength Cond Res. 2008:22(3):773-80.

11. Heggelund J, Fimland MS, Helgerud J, Hoff J. Maximal strength training improves work economy, rate of force development and maximal strength more than conventional strength training. Eur J Appl Physiol. 2013;113(6):1565-73. 
12. Hoff J, Helgerud J. Endurance and strength training for soccer players: physiological considerations. Sports Med. 2004;34(3):165-80.

13. Hoff J, Almåsbakk B. The effects of maximum strength training on throwing velocity and muscle strength in female team-handball players. J Strength Cond Res. 1995;9(4):255-8.

14. Wisløff U, Castagna C, Helgerud J, Jones R, Hoff J. Strong correlation of maximal squat strength with sprint performance and vertical jump height in elite soccer players. Brit J Sport Med. 2004;38(3):285-8.

15. Wisløff U, Helgerud J, Hoff J. Strength and endurance of elite soccer players. Med Sci Sports Exerc. 1998;30(3):462-7.

16. Behm DG, Sale DG. Intended rather than actual movement velocity determines velocity-specific training response. J Appl Physiol (Bethesda, Md : 1985). 1993;74(1):359-68.

17. Tøien T, Pedersen Haglo H, Unhjem RJ, Hoff J, Wang E. Maximal strength training: the impact of eccentric overload. J Neurophysiol. 2018;120(6):2868-76.

18. Duchateau J, Semmler JG, Enoka RM. Training adaptations in the behavior of human motor units. J Appl Physiol (Bethesda, Md : 1985). 2006;101(6):1766-75.

19. Schoenfeld BJ. The mechanisms of muscle hypertrophy and their application to resistance training. J Strength Cond Res. 2010;24(10):2857-72.

20. Bogdanis G, Papaspyrou A, Souglis A, Theos A, Sotiropoulos A, Maridaki M. Effects of a hypertrophy and a maximal strength training programme on speed, force and power of soccer players. In: The sixth World Congress on Science and Football: 2007. Antalya: Routledge; 2007. p. 290-5.

21. Schoenfeld BJ, Contreras B, Krieger J, Grgic J, Delcastillo K, Belliard R, Alto A. Resistance Training Volume Enhances Muscle Hypertrophy but Not Strength in Trained Men. Med Sci Sports Exerc. 2019;51(1):94-103. https://doi.org/1 0.1249/mss.0000000000001764.

22. Bloomquist K, Langberg H, Karlsen S, Madsgaard S, Boesen M, Raastad T. Effect of range of motion in heavy load squatting on muscle and tendon adaptations. Eur J Appl Physiol. 2013;113(8):2133-42.

23. Helgerud J, Rodas G, Kemi OJ, Hoff J. Strength and endurance in elite football players. Int J Sports Med. 2011;32(9):677-82.

24. Staron RS, Karapondo DL, Kraemer WJ, Fry AC, Gordon SE, Falkel JE, Hagerman FC, Hikida RS. Skeletal muscle adaptations during early phase of heavy-resistance training in men and women. J Appl Physiol (1985). 1994;76(3):1247-55.

25. Kell RT. The influence of periodized resistance training on strength changes in men and women. J Strength Cond Res. 2011;25(3):735-44.

26. Martel GF, Roth SM, Ivey FM, Lemmer JT, Tracy BL, Hurlbut DE, Metter EJ, Hurley BF, Rogers MA. Age and sex affect human muscle fibre adaptations to heavy-resistance strength training. Exp Physiol. 2006;91(2):457-64.

27. Haugen T, Buchheit M. Sprint running performance monitoring: methodological and practical considerations. Sports Med. 2016;46(5):641-56

28. Buckthorpe M, Morris J, Folland JP. Validity of vertical jump measurement devices. J Sports Sci. 2012;30(1):63-9.

29. Markovic G, Dizdar D, Jukic I, Cardinale M. Reliability and factorial validity of squat and countermovement jump tests. J Strength Cond Res. 2004;18(3):551-5.

30. Giorgi A, Wilson GJ, Weatherby RP, Murphy AJ. Functional isometric weight training: its effects on the development of muscular function and the endocrine system over an 8-week training period. J Strength Cond Res. 1998;12(1):18-25.

31. Arnason A, Andersen TE, Holme I, Engebretsen L, Bahr R. Prevention of hamstring strains in elite soccer: an intervention study. Scand J Med Sci Sports. 2008;18(1):40-8

32. Richardson JTE. Eta squared and partial eta squared as measures of effect size in educational research. Educ Res Rev-Neth. 2011;6(2):135-47.

33. Helgerud J, Hoff J, Wisløff U. Gender differences in strength and endurance of elite soccer players. In: Spinks W, Reilly T, Murphy A, editors. Science and Football IV. edn. Sydney: Taylor and Francis; 2002. p. 382.

34. Ahtiainen JP, Pakarinen A, Alen M, Kraemer WJ, Häkkinen K. Muscle hypertrophy, hormonal adaptations and strength development during strength training in strength-trained and untrained men. Eur J Appl Physiol. 2003:89(6):555-63.

35. Ness BM, Comstock BA, Schweinle WE. Changes in dynamic balance and hip strength after an eight-week conditioning program in ncaa division female soccer (football) athletes. Int J Sports Phys Ther. 2016;11(7):1054-64.

36. Delextrat A, Piquet J, Matthews MJ, Cohen DD. Strength-endurance training reduces the hamstrings strength decline following simulated football competition in female players. Front Physiol. 2018;9:1059.

37. Grieco CR, Cortes N, Greska EK, Lucci S, Onate JA. Effects of a combined resistance-plyometric training program on muscular strength, running economy, and Vo2peak in division I female soccer players. J Strength Cond Res. 2012;26(9):2570-6
38. Stølen T, Chamari K, Castagna C, Wisloff U. Physiology of soccer: an update. Sports Med. 2005:35(6):501-36.

39. Moritani T, deVries HA. Neural factors versus hypertrophy in the time course of muscle strength gain. Am J Phys Med Rehabil. 1979;58(3):115-30.

40. Chan ST, Johnson AW, Moore MH, Kapadia CR, Dudley HA. Early weight gain and glycogen-obligated water during nutritional rehabilitation. Hum Nutr Clin Nutr. 1982;36(3):223-32

41. Murray B, Rosenbloom C. Fundamentals of glycogen metabolism for coaches and athletes. Nutr Rev. 2018;76(4):243-59.

42. Sedano Campo S, Vaeyens R, Philippaerts RM, Redondo JC, de Benito AM, Cuadrado G. Effects of lower-limb plyometric training on body composition explosive strength, and kicking speed in female soccer players. J Strength Cond Res. 2009:23(6):1714-22.

43. Abernethy PJ, Jurimae J. Cross-sectional and longitudinal uses of isoinertial, isometric, and isokinetic dynamometry. Med Sci Sports Exerc. 1996;28(9):1180-7.

44. Voigt M, Klausen K. Changes in muscle strength and speed of an unloaded movement after various training programmes. Eur J Appl Physiol Occup Physiol. 1990;60(5):370-6.

45. Hickson RC. Interference of strength development by simultaneously training for strength and endurance. Eur J Appl Physiol Occup Physiol. 1980; 45(2-3):255-63.

46. Wilson JM, Marin PJ, Rhea MR, Wilson SM, Loenneke JP, Anderson JC Concurrent training: a meta-analysis examining interference of aerobic and resistance exercises. J Strength Cond Res. 2012;26(8):2293-307.

47. Shalfawi SA, Haugen $T$, Jakobsen TA, Enoksen $E$, Tonnessen $E$. The effect of combined resisted agility and repeated sprint training vs. strength training on female elite soccer players. J Strength Cond Res. 2013;27(11):2966-72.

48. Ozbar N, Ates S, Agopyan A. The effect of 8-week plyometric training on leg power, jump and sprint performance in female soccer players. J Strength Cond Res. 2014;28(10):2888-94.

49. Ramirez-Campillo R, Vergara-Pedreros M, Henriquez-Olguin C, MartinezSalazar C, Alvarez C, Nakamura FY, De La Fuente Cl, Caniuqueo A, AlonsoMartinez AM, Izquierdo M. Effects of plyometric training on maximalintensity exercise and endurance in male and female soccer players. $J$ Sports Sci. 2016;34(8):687-93.

50. Siegler J, Gaskill S, Ruby B. Changes evaluated in soccer-specific power endurance either with or without a 10-week, in-season, intermittent, high-intensity training protocol. J Strength Cond Res. 2003;17(2):379-87.

51. Blimkie C, J, R., Sale D: Strength development and trainability during childhood. In: Pediatric Anaerobic Performance. Edited by Van Praagh E. Champaign: Juman Kinetics; 1998: 113-224.

52. Zouita S, Zouita AB, Kebsi W, Dupont G, Ben Abderrahman A, Ben Salah FZ, Zouhal $H$. Strength training reduces injury rate in elite young soccer players during one season. J Strength Cond Res. 2016;30(5):1295-307.

53. Datson N, Hulton A, Andersson H, Lewis T, Weston M, Drust B, Gregson W. Applied physiology of female soccer: an update. Sports Med. 2014;44(9):1225-40.

54. Nikolaidis PT. Weight status and physical fitness in female soccer players: is there an optimal BMI? Sport Sci Health. 2014;10(1):41-8.

\section{Publisher's Note}

Springer Nature remains neutral with regard to jurisdictional claims in published maps and institutional affiliations.

Ready to submit your research? Choose BMC and benefit from:

- fast, convenient online submission

- thorough peer review by experienced researchers in your field

- rapid publication on acceptance

- support for research data, including large and complex data types

- gold Open Access which fosters wider collaboration and increased citations

- maximum visibility for your research: over $100 \mathrm{M}$ website views per year

At BMC, research is always in progress.

Learn more biomedcentral.com/submissions 